\title{
AngioJet-assisted transvenous-transhepatic mechanical thrombectomy in the portal vein
}

\author{
Daniel Kuetting ${ }^{A, B, D, E, F}$, Karsten Wolter ${ }^{B}$, Julian Luetkens ${ }^{B, F}$, Jonel Trebicka ${ }^{D, E}$, Michael Praktiknjo ${ }^{D, E}$, \\ Daniel Thomas ${ }^{\mathrm{B}, \mathrm{D}}$, Carsten Meyer ${ }^{\mathrm{A}, \mathrm{B}, \mathrm{D}, \mathrm{E}}$ \\ University of Bonn, Germany
}

\section{Abstract}

Purpose: To evaluate AngioJet-assisted transvenous portal vein (PV) thrombectomy for non-cirrhotic patients with total portal vein and mesenteric vein thrombosis (PVMVT).

\begin{abstract}
Material and methods: From 2015 to 2016 four patients ( 3 male, mean 43.9 years, range 33-52 years) with acute ( 3 cases) and acute-on-chronic (1 case) PVMVT underwent transvenous thrombolysis. All patients received initial AngioJet (Boston Scientific, Natick, MA, USA) thrombectomy followed by continuous catheter directed thrombolysis with Urokinase (Medac, Wedel, Germany) for 22-52 hours. Transjugular intrahepatic portosystemic shunts (TIPS), using Viatorr stent grafts (W.L. Gore and Associates, AZ, USA; mean diameter: $10 \mathrm{~mm}$, length: 60-80 mm), were implanted in all patients. Patients were followed clinically and with imaging (mean 646 days, range 392 to 936 days).

Results: Technical success was $100 \%$. Therapeutic success was achieved in $75 \%$ of cases. AngioJet-assisted thrombectomy substantially reduced thrombus load in the acute cases, while only slight improvement was achieved in the acuteon-chronic case. Continuous thrombolysis subtotally re-established PV flow in the acute cases, while only minimal improvement was seen in the acute-on-chronic case. Following TIPS implantation complete PV recanalisation could be achieved in all acute cases. In the acute-on-chronic case initial stagnant PV flow was seen; however, PV and TIPS re-occluded 10 days after implantation. During follow-up PV remained patent in acute cases.

Conclusions: AngioJet-assisted thrombectomy was technically feasible and uncomplicated in all of our patients. The initial results suggest that AngioJet-assisted thrombectomy facilitates recanalisation in acute and severe cases of PVMVT.
\end{abstract}

Key words: portal vein thrombosis, thrombectomy, trans jugular portosystemic shunt.

\section{Introduction}

In the general population acute portal vein thrombosis (PVT) is a rare disease [1]; however, among patients with cirrhosis its prevalence is as high as $15 \%$ [2], accompanied by significantly increased mortality rates [3]. In noncirrhotic patients PVT is infrequent and commonly caused by myeloproliferative diseases or congenital coagulopathies. Nevertheless, non-cirrhosis-associated PVT is of growing interest; it is linked with increased morbidity and markedly shortened life expectancy in a commonly young and typically otherwise healthy patient collective [4].

Intestinal infarction with perforation and peritonitis are the most troubling immediate complications of PVT, with a related mortality rate of up to $60 \%$. Thus, severe PVT is a medical emergency and necessitates rapid therapy. Successful recanalisation of acute PVT following anticoagulative monotherapy can be achieved in $38 \%$ of acute cases, while success is less probable in chronic and severe cases of PVT $[5,6]$. Endovascular, catheter-directed thrombolytic

Correspondence address:

Dr. Daniel Kuetting, University of Bonn, Sigmund Freud Strasse 25, 531277, Bonn, Germany, phone: +4915162315431, e-mail: daniel.kuetting@ukbonn.de

Authors' contribution:

A Study design · B Data collection · C Statistical analysis · D Data interpretation · E Manuscript preparation · F Literature search · G Funds collection 

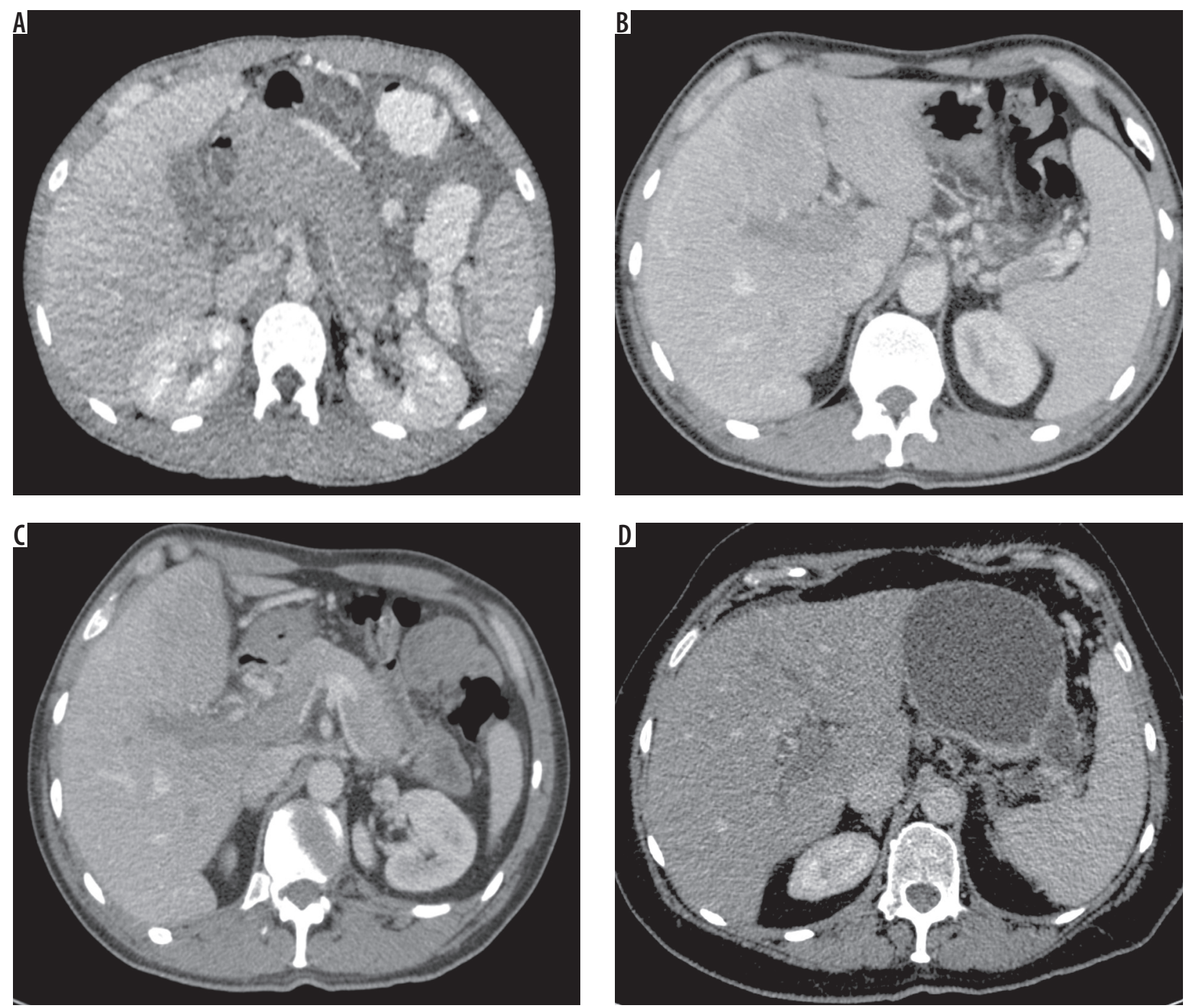

Figure 1. Computed tomography (CT) images acquired in portal venous phase of a 33-year-old male - case 1 (A), 47-year-old male - case 2 (B), and a 44-year-old male - case 3 (C) show acute and extensive portal venous thrombosis. (T images of a 52-year-old female - case 4 (D) - show acuteon-chronic portal vein thrombosis

therapy is an effective therapeutic option, supported by previously conducted studies reporting good results $[7,8]$ and has now been adopted in the guidelines for treatment of PVT [9]. In theory portal vein (PV) thrombectomy allows for more rapid recanalisation of the PV.

Although several endovascular devices have been suggested for device-assisted PV-thrombectomy [10-13], data regarding the efficacy of this procedure are still sparse. We sought to investigate the efficacy of the AngioJet (Boston Scientific, Natick, MA, USA) for the treatment of massive portal vein and mesenteric vein thrombosis (PVMT). We present four cases of acute and acute-on-chronic PVMT in which restoration of PV patency was attempted by transjugular, transhepatic, AngioJet-assisted mechanical thrombectomy followed by establishment of TIPS.

\section{Material and methods}

Four patients (one female, mean age 43.9 years, range 3352 years) with, respectively, acute, acute-on-chronic portal vein, and mesenteric vein thrombosis (PVMVT) underwent transvenous PV thrombectomy between 2015 and 2016, in order to re-establish PV patency.

All patients were surveyed clinically or by telephone follow-up and with imaging (mean 646, range 392 days to 936 days). None of the patients had a history of liver cirrhosis.

All patients gave their written consent that clinical history, image material, and laboratory results could be used for scientific purposes. This study was carried out in accordance with the International Ethical Guidelines and the Declaration of Helsinki.

Technical success was defined as uncomplicated transjugular-transhepatic access to the PV system combined with uncomplicated implementation of the AngioJet device for thrombectomy. Therapeutic success was defined as recanalisation of the PV, splenic vein, superior mesenteric vein (SMV), and inferior mesenteric vein (IMV) with re-established hepatopetal flow following transvenous-transhepatic AngioJet-assisted PV 
thrombectomy and subsequent catheter directed continuous lysis.

\section{Material}

\section{Case 1}

A 33-year-old male presented with a seven-day history of progressive epigastric pain as well as new onset, slightly bloody diarrhoea. The patient had no history of bloody stools or similar abdominal pain in the past. Physical examination revealed severe abdominal tenderness and decreased bowel activity. The patient was haemodynamically stable. Initial laboratory study revealed elevated C reactive protein (CRP) of $120 \mathrm{mg} / 1$ [normal range: $0-3 \mathrm{mg} / \mathrm{l}]$. Lactate was not increased with $1.10 \mathrm{mmol} / \mathrm{l}$. Bilirubin $(1.98 \mathrm{mg} / \mathrm{dl})$ [normal range: $0.1-1 \mathrm{mg} / \mathrm{dl}$ ], alkaline phosphatase (141 U/l) [normal range: 34-117 U/l] and aminotransferase (ALT $58 \mathrm{U} / \mathrm{l}$ ) [normal range: $<50 \mathrm{U} / \mathrm{l}]$ were slightly elevated. Otherwise laboratory results were within normal range.

Computed tomography (CT) (Figure 1A) revealed extensive PVMVT with complete thrombotic occlusion of the intrahepatic PV. The extrahepatic PV $(>4 \mathrm{~cm})$, the splenic vein $(3.3 \mathrm{~cm})$, the SMV $(4.6 \mathrm{~cm})$, and the IMV $(3.6 \mathrm{~cm})$ showed subtotal thrombotic occlusion extending into the pelvis and were markedly distended. Intestinal wall swelling of the small bowel was evident without signs of pneumatosis intestinalis.

\section{Case 2}

A 47-year-old male with a history of recurring deep vein thrombosis for which the patient had received warfarin for six months; otherwise the patient had no significant past medical history. Initial laboratory study revealed an increased level of inflammatory indicators (leukocytes $11.4 \mathrm{G} / \mathrm{l}$, CRP $27.2 \mathrm{mg} / \mathrm{l}$ ) and a slightly elevated aminotransferase (ALT $61 \mathrm{U} / \mathrm{l}$ ); lactate was not increased, at $1.20 \mathrm{mmol} / \mathrm{l}$. Otherwise laboratory results were inconspicuous.

The CT study (Figure 1B) revealed extensive PVMVT with complete thrombotic occlusion of the intrahepatic $\mathrm{PV}$, and total thrombotic occlusion of the extrahepatic $\mathrm{PV}$, the splenic vein, the SMV, and the IMV. Additionally, a mosaic pattern of hepatic perfusion was noted. The extrahepatic PV $(2 \mathrm{~cm})$ and the SMV $(1.8 \mathrm{~cm})$ were marginally dilated. CT showed no signs of intestinal ischaemia.

\section{Case 3}

A 44-year-old male with known protein-S deficiency and history of deep vein thrombosis. The patient had stopped taking his prescribed Rivaroxaban due to recurrent haemorrhoidal bleeding. The patient presented with progressive abdominal tenderness. Initial laboratory findings showed an increased level of inflammation (CRP $54.4 \mathrm{mg} / \mathrm{l})$. Lactate was not increased, at $1.1 \mathrm{mmol} / \mathrm{l}$. Alkaline phosphatase (122 U/l) and aminotransferase (ALT $57 \mathrm{U} / \mathrm{l}$ ) were slightly elevated. Otherwise laboratory results were inconspicuous.

CT (Figure 1C) demonstrated total occlusion of the main PV, the splenic vein, the SMV, and IMV, while intrahepatic portal veins were mainly patent. Additionally, mesenteric stranding could be seen, especially surrounding the jejunum. Otherwise no signs of intestinal ischaemia were evident.

\section{Case 4}

A 52-year-old female with a known history of recurrent pancreatitis and partial PV thrombosis, who presented with acute abdominal pain and signs of ileus. Initial laboratory study revealed an increased level of inflammation (leukocytes $38.25 \mathrm{G} / \mathrm{l}$, CRP $186.0 \mathrm{mg} / \mathrm{l}$ ) and slightly elevated gamma GT (38 U/l) [normal range: > $55 \mathrm{U} / 1$ ], lactate was not increased, at $1.30 \mathrm{mmol} / \mathrm{l}$. Otherwise laboratory results were inconspicuous. Ultrasound showed moderate amounts of ascites. Paracentesis revealed spontaneous bacterial peritonitis with 2068 granulocytes/ $\mu \mathrm{l}$, which was treated antibiotically.

CT (Figure 1D) demonstrated total obstruction of the PV, as well as partial occlusion of the splenic vein, the SMV, and IMV, with large amounts of wall-adherent thrombotic material, which led to the diagnosis of an acute-on-chronic PV thrombosis. Also, signs of chronic pancreatitis without acute oedema, a $3.5 \mathrm{~cm}$ large pseudocyst, moderate amounts of ascites, as well as signs of paralytic ileus were diagnosed.

\section{Procedure}

Because symptoms progressed despite anticoagulation (intravenous heparin, partial thromboplastin time - PTT in therapeutic range), all patients were referred to interventional PV-thrombolysis/thrombectomy following interdisciplinary case discussion. All procedures were carried out following informed consent and detailed review of previous imaging. The procedures were performed using aseptic techniques under local anaesthesia ( $1 \%$ lidocaine), intravenous analgesia, and sedation (pethidine and midazolam).

After attaining ultrasound-guided jugular venous access, a $10 \mathrm{~F}$ sheath (Terumo, Tokyo, Japan) was inserted. The right hepatic vein was catheterised with a $4 \mathrm{~F}$ multipurpose catheter. The portal venous system was accessed transhepatically under ultrasound guidance with the use of $16 \mathrm{G}$ transjugular liver access and biopsy needle set (Cook, Bjaeverskov, Denmark). A hydrophilic-coated guidewire and a $4 \mathrm{~F}$ multipurpose catheter were then manipulated through the occluded portal vein into the SMV. Direct portography was performed to document the extent of 

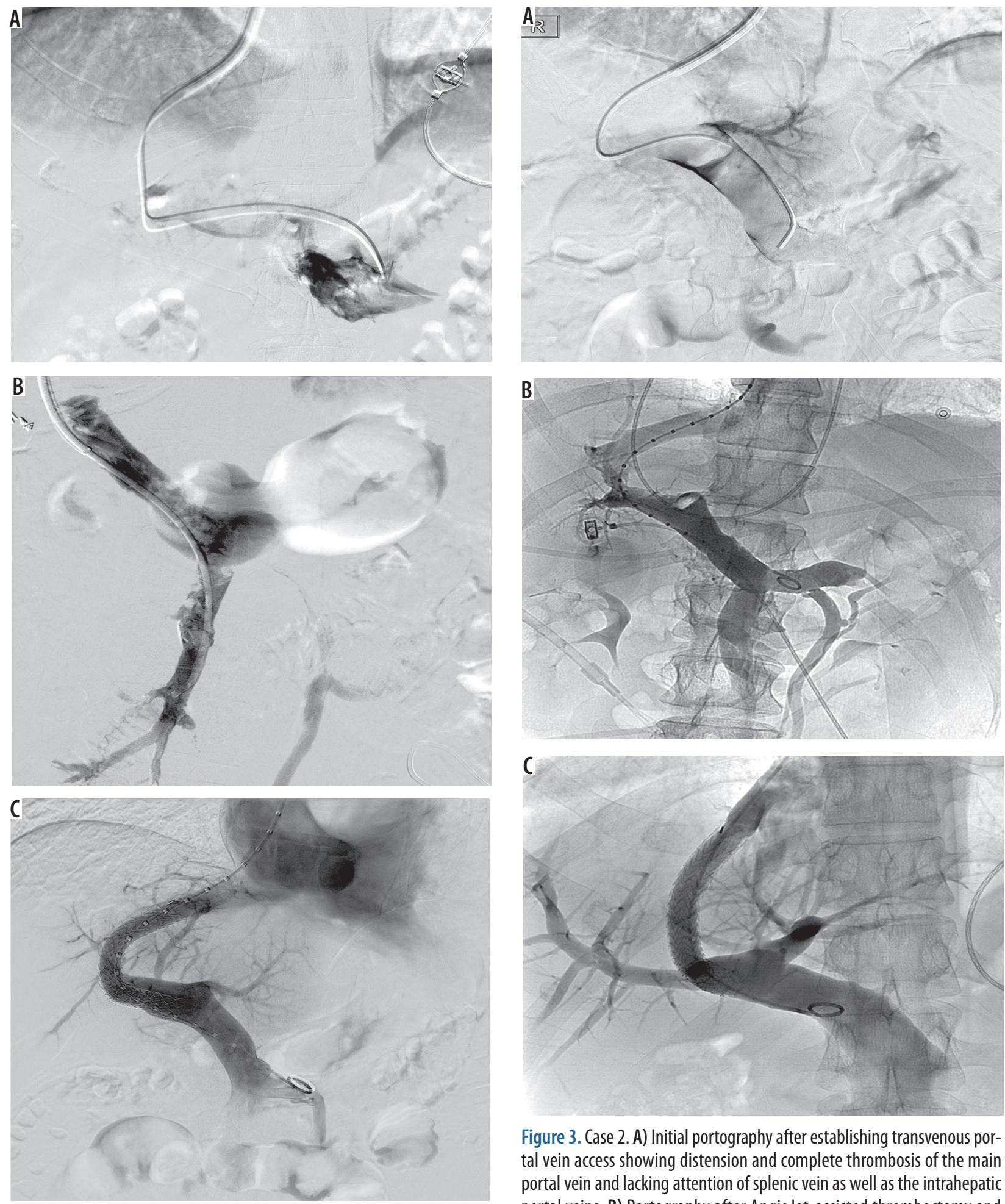

Figure 3. Case 2. A) Initial portography after establishing transvenous portal vein access showing distension and complete thrombosis of the main portal vein and lacking attention of splenic vein as well as the intrahepatic portal veins. B) Portography after AngioJet-assisted thrombectomy and 22 hours of catheter-directed thrombolysis therapy showing subtotal reso-

Figure 2. Case 1. A) Initial portography after establishing transvenous portal vein access showing complete thrombosis of the portal vein without attention of intrahepatic portal veins. B) Portography after AngioJet-assisted thrombectomy and 46 hours of catheter-directed thrombolysis therapy, showing residual amounts of thrombus in the superior mesenteric vein and the main portal trunk. C) Portography following establishment of transjugular intrahepatic portosystemic shunts showing complete recanalisation of the portal vein

the thrombotic occlusion (Figures 2-4). The guidewire was then exchanged for a PTFE-covered, stiff guidewire with a stainless-steel core (Amplatz, Cook, Bjaeverskov, Denlution of portal vein and mesenteric vein thrombosis but residual intrahepatic portal vein (PV) thrombus. C) Portography following establishment of transjugular intrahepatic portosystemic shunts showing complete recanalisation of the PV

mark). The short $10 \mathrm{~F}$ sheath was removed in exchange for a long $10 \mathrm{~F}$ sheath (Check-Flo Performer Introducer, Cook, Bjaeverskov/Denmark) and advanced into the PV after pre-dilatation of the puncture tract with 6-8-mm balloons (Mustang, Boston Scientific, Natick, MA, USA). 

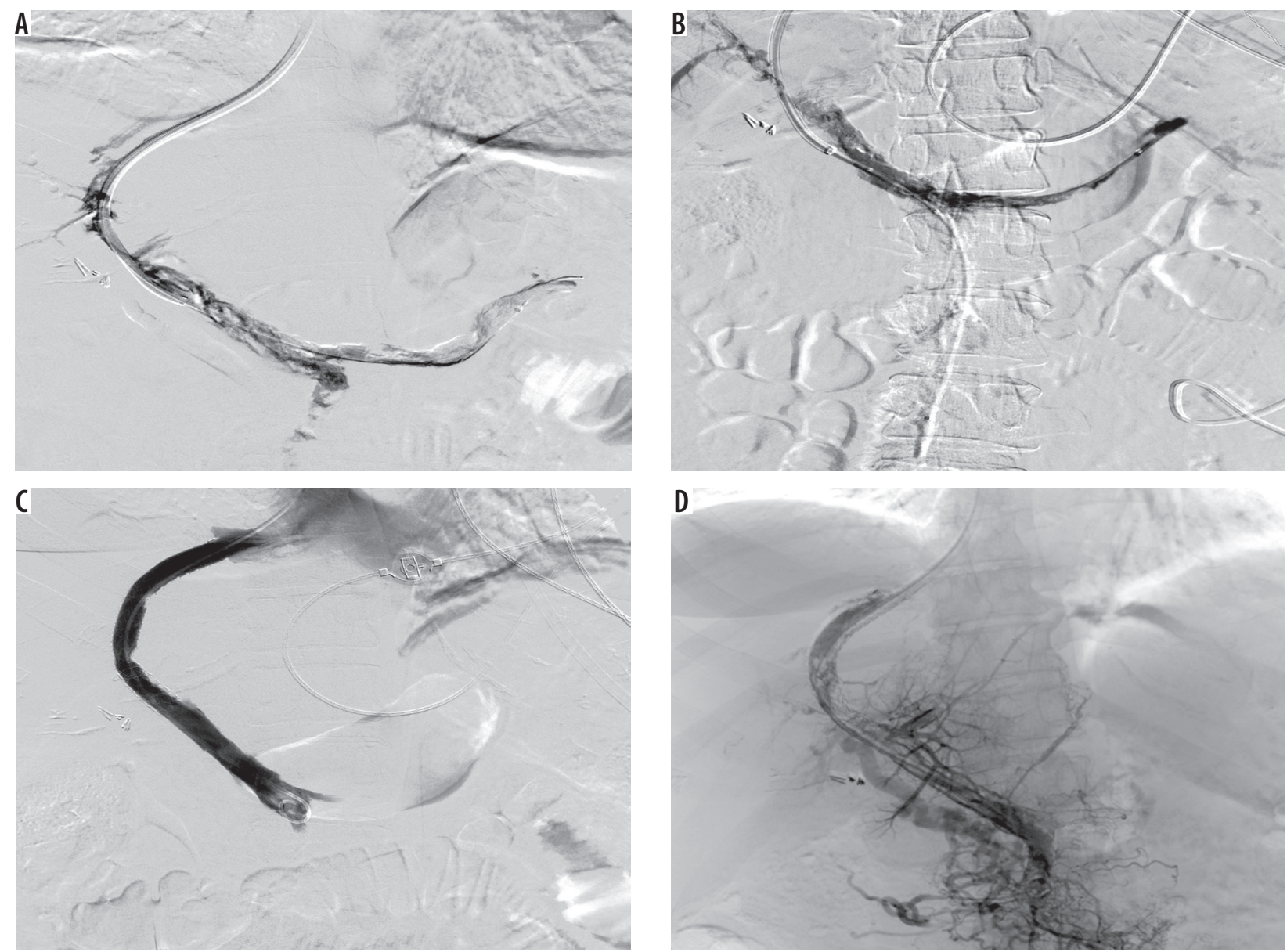

Figure 4. (ase 4. A) Initial portography after establishing transvenous portal vein access showing lacking attenuation of the intrahepatic portal veins and subtotal thrombosis of the main portal vein, the splenic vein, and the superior mesenteric vein. B) Portography after AngioJet-assisted thrombectomy and 48 hours of catheter-directed thrombolysis therapy showing only minimally improved portal venous flow and persisting complete thrombosis of the intrahepatic portal veins. C) Portography following establishment of transjugular intrahepatic portosystemic shunts (TIPS) showing recanalisation of the main portal vein (PV) but lacking attenuation of the intrahepatic portal veins, the splenic vein, and the superior mesenteric vein. D) Repeat portography performed 10 days after establishment of TIPS showing significant thrombotic re-occlusion of the PV and partial thrombosis of the TIPS

Using the spray function of the AngioJet thrombectomy device (Boston Scientific, Natick, MA, USA) the thrombus in the splenic vein, the inferior mesenteric vein, the superior mesenteric vein, and the main portal vein were pre-treated with 250,000 IU of Urokinase (Medac, Wedel, Germany) diluted in saline. After 15 minutes of dwell time allowing for initial thrombus dissolution, mechanical thrombectomy was performed using the power-pulse spray technique. Large amounts of red thrombus could be harvested in cases 1-3, while only small amounts of mainly white thrombus could be harvested in case 4 .

In the first three cases control portography revealed severely reduced thrombus load with persistent evidence of flow-limiting clot (Figures 2 and 3). In the fourth case, only a slight reduction of thrombus load was seen (Figure 4). Extravasation was not seen in any of the cases. Subsequent catheter-directed thrombolysis was performed in all cases due to residual PV thrombosis. Multiple-side-hole infusion catheters (Uni Fuse, Angiodynamics, NY, USA) were inserted into the SMV and/or splenic vein/IMV over which continuous, catheter-directed thrombolysis was applied for 22-52 hours with a high dose of uroki- nase $(100,000 \mathrm{IU} / \mathrm{h})$. After restoring hepatopetal venous flow in cases 1-3 (Figures 2,3) a transjugular intrahepatic portosystemic shunt (TIPS) was implanted to completely re-establish and preserve PV patency. In case 4 TIPS was implanted as a bailout solution because thrombolytic therapy as well as additional aspiration thrombectomy led to only minimal improvement of PV-flow (Figure 4).

In all cases polytetrafluoroethylene-covered stent-grafts (Viatorr; W.L. Gore and Associates, AZ, USA) were used. All stents grafts had a nominal diameter of $10 \mathrm{~mm}$. Stent lengths were chosen according to the distance of the confluence of the hepatic veins to the site of PV access. The employed stent lengths were 60, 70, and $80 \mathrm{~mm}$ (Figures 2-4). Post-placement stent-graft dilatation was not performed. Postinterventional PTT-adapted heparinisation was continued in all cases for at least 24 hours (goal PTT $40-60 \mathrm{sec}$ ), followed by oral anticoagulation with vitamin $\mathrm{K}$ antagonists.

\section{Results}

Technical success was achieved in all four cases. Therapeutic success was achieved in three out of four in cases. 
Urokinase lysis therapy was performed until hepatopetal flow was re-established in the PV, the splenic vein, the SMV, and the IMV. Lytic therapy was performed for 46 (case 1), 22 (case 2), and 27 hours (case 3), leading to a total dose of 3,650,000 IU (case 1), 2,050,000 IU (case 2), and 2,950,000 IU (case 3).

Following the establishment of TIPS, complete recanalisation of the PV, the splenic vein, the SMV, and the IMV were documented by angiography and by sonography in cases 1-3 (Figures 2 and 3).

In case $4 \mathrm{PV}$ thrombosis was almost completely refractory to the intervention and also to subsequent catheter-directed continuous lysis. Because control angiography showed only minimal signs of improvement (Figure 4) thrombolytic therapy was stopped after 52 hours of continuous urokinase administration (applied total dose: 5,200,000 IU). Following the establishment of TIPS, partial recanalisation of the main PV was achieved; however, PV flow remained stagnant. The intrahepatic portal vein branches, the splenic vein, the SMV, and the IMV remained mainly occluded (Figure 4).

\section{Clinical symptoms}

In cases 1 to 3 symptomatic relief of abdominal pain and or bloody diarrhoea was attained before discharge. In case 4 peritonitis and its complications (paralytic ileus) were successfully treated, and abdominal pain could be reduced, but it persisted until discharge.

\section{Complications}

Cases 1 and 2 developed temporary haematuria during thrombolytic therapy, which did not lead to bladder tamponade, did not affect haemoglobin levels, and did not necessitate transfusion. Urokinase dosage was reduced to 50,000 IU/h, and three-way Foley urinary catheters were introduced. Otherwise, no immediate complications were identified.

\section{Screening}

Thrombophilia screening was performed parallel to endovascular thrombectomy/thrombolysis in all patients. In case 1 screening revealed no conspicuous findings. In cases 2 and 4 a JAK2-V617F-mutation, consistent with myeloproliferative syndrome, was diagnosed. In case 3 a protein-S deficiency had been pre-diagnosed, and thrombophilia screening revealed no further abnormalities. Shear-wave elastography revealed no signs of underlying liver cirrhosis in any of the cases.

\section{Follow up}

Case 1: During follow-up the patient presented with an episode of encephalopathy four months after TIPS im- plantation, after having discontinued his laxatives a week before. Encephalopathy could be controlled medically. In following controls (total duration: 31 months) ultrasound revealed PV and TIPS patency with intrahepatic PV hepatopetal flow. Follow-up laboratory tests showed normal values of liver enzymes, and follow-up psychometric tests revealed no signs of reoccurrence of encephalopathy.

Case 2: During follow-up controls (total duration: 24 months) ultrasound revealed PV and TIPS patency with intrahepatic PV hepatopetal flow. Follow-up laboratory tests showed normal values of liver enzymes. Psychometric tests revealed no signs of encephalopathy.

Case 3: Ultrasound follow-up two months post intervention showed that TIPS and PV were patent. MRI performed three months post procedure, however, revealed TIPS occlusion with persistent PV patency. The reason for TIPS thrombosis remained unclear. Recanalisation was not attempted because PV remained patent, and the patient remained clinically stable. Further clinical controls (total duration nine months) with laboratory and ultrasound follow-up confirmed PV patency and showed normal values of liver enzymes. Psychometric tests revealed no signs of encephalopathy. The patient did not appear to further clinical examinations. During telephone follow-up performed 17 months post-procedure the patient reported having neither abdominal symptoms nor subjectively noticeable cognitive impairment.

Case 4: Ultrasound performed 10 days after implantation of TIPS revealed subtotal thrombotic TIPS occlusion (Figure 4). The patient was referred to interventional radiology again to attempt TIPS- and PV-recanalisation. By means of aspiration thrombectomy and thrombolytic therapy, PV and TIPS were successfully recanalised; however, the TIPS re-occluded again 10 days later. Another attempt to re-establish PV patency was not undertaken.

Duplex sonography performed two months and 12 months after intervention showed a complete occlusion of the TIPS tract and the splanchnic venous system with cavernous transformation of the portal vein. Gastroscopy revealed oesophageal varices $I^{\circ}$, psychometric testing revealed no signs of hepatic encephalopathy, and laboratory follow-up showed normal values of liver enzymes.

\section{Discussion}

The management of acute PVT in patients without cirrhosis or malignancy is complex and not yet sufficiently represented in national guidelines. Recently, however, the consensus paper of the Baveno VI conference [9] was released, on which the European guidelines of the European Association for the Study of the Liver (EASL) are based. The consensus paper includes revised treatment recommendations of non-cirrhotic PVT: Initial therapy is identical to that of cirrhotic PVT, with immediate commencement of low-molecular-weight heparin followed by oral anticoagulant therapy. In the case of persistent abdominal 
pain, varicose haemorrhage, bloody diarrhoea, or lactic acidosis despite commencement of anticoagulative therapy [9], interventional revascularisation should be considered in non-cirrhotic PVT patients. The therapy of choice to achieve revascularisation, however, is not specified.

In this regard, several different interventional techniques (systemic, indirect, and direct) have been proposed to access and administer thrombolytic agents in the PV. Among these the direct approaches appear the most promising, with higher success rates in comparison to systemic and indirect approaches [13]. Another advantage of the direct approach is the prospect of device-assisted thrombectomy/thrombolysis, which has been shown to expedite the procedure $[11,12]$.

Although the duration of thrombolytic therapy in the current study was comparable to prior studies and case reports where only catheter directed pharmacological lysis was performed [14-16], the extent of PV and splanchnic thrombosis was more substantial in the current cases. We therefore believe that AngioJet-assisted thrombectomy expedited the procedure nonetheless.

Although randomised control trials regarding the efficacy of minimally invasive direct PV thrombolysis are still lacking, the published case reports and case series [10,16-18], now including over 150 patients, indicate promising results, with recanalisation rates as high as $80 \%$ [6].

Direct thrombolysis/thrombectomy of the PV is feasible via a percutaneous trans-splenic-, percutaneous transhepatic, as well as a transvenous transhepatic approach. In a recently published case series including five patients the combination of the trans-splenic and the transhepatic approach showed good results (100\% technical success rate) for treatment of chronic PVT [10]. However, the authors concede that there are potential complications of splenic capsular perforation and abdominal bleeding. Similarly, the percutaneous transhepatic approach $[8,11]$ also requires capsule perforation and is therefore associated with an elevated risk of complications (up to $60 \%$ of patients show subcapsular hepatic haematoma or intra-abdominal bleeding) $[8,16]$. In comparison, the transvenous-transhepatic approach also allows for direct thrombolysis without obligatory organ capsule perforation and allows for post-lysis TIPS establishment to ensure PV patency [19]. Because the transvenous-transhepatic approach (unlike the two above-mentioned techniques) does not usually pass through the intraperitoneal cavity, it is also suitable for patients with existing ascites and coagulative dysfunction [15].

In theory, percutaneous mechanical thrombectomy/ thrombolysis devices can decrease the duration of lytic therapy and reduce the required amount of thrombolytic drugs. Several different devices have been successfully used for portal vein thrombectomy/thrombolysis; among these are the Trellis, the EKOS, and the AngioJet device.

The Trellis device (Covidien, Bacchus Vascular, CA, USA), which is currently not commercially available, con- sists of an oscillating nitinol wire between two balloons between which thrombolytic agents can be injected. In a case reported by Darcy an occluded TIPS and thrombosed PV could be recanalised with the Trellis device via a trans-jugular approach in combination with thrombolysis therapy in a single session [12]. The patient developed postinterventional diffuse alveolar haemorrhage, which necessitated intubation and bronchoscopy. In following ultrasound controls TIPS and PV remained patent.

The ultrasound-assisted EKOS Endowave device (EKOS Corporation, WA, USA) is a multiple side-hole infusion catheter, which additionally contains ultrasound transducers that emit low power sound waves. These sound waves cause fibrin strands to thin, thus expediting lysis. Abdel Aal et al. recently presented a case in which ultrasound-accelerated PV thrombolysis performed in a patient with acute, partial thrombosis via a transhepatic approach could severely reduce thrombus load after 30 hours of ultrasound-accelerated thrombolysis [11]. $\mathrm{PV}$ remained patent in the following controls, and no complications associated with the intervention were noted.

The AngioJet device combines pharmacological and mechanical thrombectomy. In the first step a spray engine is used to disperse the lytic agent throughout the thrombus. After letting the lytic agent dwell for several minutes, the spray engine is combined with a high-pressure pump, which leads to an intra-vasal pressure gradient (Venturi effect), ultimately causing thrombus fragmentation and allowing aspiration of thrombus into the catheter.

Although AngioJet-assisted transvenous-transhepatic portal venous thrombectomy followed by the creation of TIPS has been successfully used in previous case reports $[20,21]$, to date it is not an established therapy option. The current results support that the AngioJet device can effectively reduce thrombus load in the setting of acute PVT. In three of our cases thrombus load could be significantly reduced, leading to a reduction of both the duration of the subsequent continuous thrombolytic therapy and the amount of applied thrombolytic agent.

In the fourth case of our series AngioJet therapy could only minimally reduce PV-thrombus load, and also continuous thrombolytic therapy showed no significant improvement of PV-flow. This patient had a known history of partial PV-thrombosis and probably presented with an acute-on-chronic PV thrombosis, highlighted by the fact that mechanical thrombectomy retracted mainly white thrombus and only small amounts of red thrombus from the PV. Even though endovascular approaches have been shown to enable successful recanalisation of chronic PV thrombosis [10], the transvenous-transhepatic approach using the AngioJet does not appear favourable for this intervention.

It is debatable whether mechanical thrombectomy should be performed initially or following primary continuous thrombolytic therapy. Both approaches offer advantages. We chose to initiate therapy with mechanical 
thrombectomy. Removing thrombus load increases the surface of the remaining thrombus, consequently making thrombolytic therapy more effective. Performing initial continuous thrombolysis will possibly facilitate mechanical thrombectomy; however, it may also significantly increase the risk of haemorrhage during the intervention.

Contrary to Haskal's report [20], in which AngioJet thrombectomy sufficed to recanalise a thrombosed $\mathrm{PV}$, in the current cases continuous lysis had to be performed following initial AngioJet-assisted thrombectomy. Although the extent of PV thrombosis was not specified in the case by Haskal, we presume that additional continuous lytic therapy was necessary in the current patient collective due to more severe PV thrombosis (thrombosis extending into the pelvis, markedly dilated PV and mesenteric veins).

Following successful recanalisation of the PV, implantation of TIPS should be discussed in cases with extensive PV thrombosis as well as residual clot following lytic therapy. Once hepatopetal PV-flow is re-established, creation of a portosystemic shunt can increase splanchnic flow and thus generate a sufficient gradient to expedite residual clot dissolution and maintain PV patency, as could be seen in three of our four patients. Furthermore, TIPS decreases the risk of PV re-thrombosis [22]. In the fourth patient TIPS was implanted as a bailout solution in an attempt to improve PV-flow because lytic therapy had failed to re-establish hepatopetal flow. However, insufficient inflow may lead to occlusion/re-occlusion of PV and TIPS. Therefore, TIPS implantation should only be advocated in PV-thrombosis patients in whom hepatopetal PV flow has been re-established.

Concordant with the report of Haskal [20], TIPS was established only after having performed device-assisted PV-thrombectomy. Although this does not eliminate the risk of peri-interventional pulmonary embolisation (PE), it is markedly reduced nonetheless, further highlighted by the fact that PE was not clinically evident in any of the included patients nor reported in the case by Haskal.

Careful patient selection is essential because TIPS can lead to a deterioration of liver function and hepatic encephalopathy [23], even in the absence of primary liver disease. Although PVT was previously considered a contraindication for TIPS [24], recent results from centres specialising in TIPS demonstrate that patients with severe acute and chronic PVT tend to profit from a portosystemic shunt [25-27]. Currently, data is still lacking regarding whether TIPS should be occluded or remain patent after successful PV recanalisation. In the current cases the interdisciplinary decision was made to not occlude TIPS due to the elevated risk of re-thrombosis in this patient collective. It is important to note that not every patient with PVT requires the implantation of TIPS. However, TIPS has been shown to increase PV patency and decrease mortality in patients with severe PVT and life-threatening complications of portal vein thrombosis as well as congenital/acquired coagulopathies with increased risk of re-thrombosis [28].
In our patient collective TIPS were implanted because of extensive PVT involving both the SMV and the IMV, and it was also indicated by prothrombotic predisposition in two of these.

Follow-up and complete diagnostic work-up of PVT is just as important as acute treatment.

Patients who do not show signs of cirrhosis or who present with compensated cirrhosis (Child A or B) should be thoroughly evaluated for conditions that may predispose PVT. Thrombophilic conditions or other triggering factors can be identified in up to $75 \%$ of cases [29]. Diagnosing the underlying aetiological factor for PVT may have a therapeutic impact; for instance, the presence of prothrombotic disorders influences the duration of anticoagulant treatment in PVT - these have been shown to be independent predictors of recurrent thrombosis [30]. In our patient collective all patients, including the patient lacking a coagulopathy, received indefinite oral anticoagulation with vitamin $\mathrm{K}$ antagonists, due to the extent of thrombosis and the risk of PV re-thrombosis.

There are limitations to our study that have to be discussed. We conducted this study retrospectively in a small patient collective, owing to the fact that PVT is a rather rare entity in non-cirrhotic patients. Nevertheless, the high rate of successful recanalisation combined with a low complication rate is promising.

In summary, the goal of therapy is a rapid recanalisation of the PV and SMV/IMV to prevent venous infarction and portal hypertension. In addition to firstline therapy (anticoagulation), the device-assisted transvenous-transhepatic approach has been shown to be effective and safe with high rates of recanalisation. AngioJet-assisted thrombectomy was technically feasible and uncomplicated in all of our patients. The initial results suggest that AngioJet-assisted thrombectomy combined with thrombolysis enables recanalisation in acute and severe cases of PVMVT.

\section{Conclusions}

Transvenous transhepatic AngioJet-assisted recanalisation of acute portal vein thrombosis is a safe and effective approach.

AngioJet-assisted thrombectomy appears not to be favourable in chronic portal vein thrombosis.

Establishment of transjugular intra-hepatic stent shunt (TIPS) following PV recanalisation can help preserve PV patency.

\section{Conflict of interest}

The authors report no conflict of interest. 


\section{References}

1. Rajani R, Bjornsson E, Bergquist A, et al. The epidemiology and clinical features of portal vein thrombosis: a multicentre study. Aliment Pharmacol Ther 2010; 32: 1154-1162.

2. Kinjo N, Kawanaka H, Akahoshi T, et al. Portal vein thrombosis in liver cirrhosis. World J Hepatol 2014; 6: 64-71.

3. Stine JG, Shah PM, Cornella SL, et al. Portal vein thrombosis, mortality and hepatic decompensation in patients with cirrhosis: A meta-analysis. World J Hepatol 2015; 7: 2774-2780.

4. Janssen HL, Wijnhoud A, Haagsma EB, et al. Extrahepatic portal vein thrombosis: aetiology and determinants of survival. Gut 2001; 49: 720-724.

5. Sogaard KK, Astrup LB, Vilstrup H, et al. Portal vein thrombosis; risk factors, clinical presentation and treatment. BMC Gastroenterol 2007; 7: 34

6. Plessier A, Darwish-Murad S, Hernandez-Guerra M, et al. Acute portal vein thrombosis unrelated to cirrhosis: a prospective multicenter follow-up study. Hepatology 2010; 51: 210-218.

7. Lopera JE, Correa G, Brazzini A, et al. Percutaneous transhepatic treatment of symptomatic mesenteric venous thrombosis. J Vasc Surg 2002; 36: 1058-1061.

8. Kim HS, Patra A, Khan J, et al. Transhepatic catheter-directed thrombectomy and thrombolysis of acute superior mesenteric venous thrombosis. J Vasc Interv Radiol 2005; 16: 1685-1691.

9. de Franchis R. Expanding consensus in portal hypertension: Report of the Baveno VI Consensus Workshop: Stratifying risk and individualizing care for portal hypertension. J Hepatol 2015; 63: 743-752.

10. Kallini JR, Gabr A, Kulik L, et al. Noncirrhotic complete obliterative portal vein thrombosis: Novel management using trans-splenic transjugular intrahepatic portosystemic shunt with portal vein recanalization. Hepatology 2016; 63: 1387-1390.

11. Abdel-Aal AK, Ezzeldin IB, Hamed MF, et al. Endovascular treatment of acute portal vein thrombosis using ultrasound-accelerated catheter-directed thrombolysis. Vasc Endovascular Surg 2014; 48 : 460-465.

12. Darcy M. Single-session treatment of portal vein thrombosis using combined pharmacomechanical thrombolysis. Semin Intervent Radiol 2007; 24: 327-332.

13. Liu FY, Wang MQ, Fan QS, et al. Interventional treatment for symptomatic acute-subacute portal and superior mesenteric vein thrombosis. World J Gastroenterol 2009; 15: 5028-5034.

14. Henao EA, Bohannon WT, Silva MB, Jr. Treatment of portal venous thrombosis with selective superior mesenteric artery infusion of recombinant tissue plasminogen activator. J Vasc Surg 2003; 38 1411-1415.

15. Aytekin C, Boyvat F, Kurt A, et al. Catheter-directed thrombolysis with transjugular access in portal vein thrombosis secondary to pancreatitis. Eur J Radiol 2001; 39: 80-82.
16. Hollingshead M, Burke CT, Mauro MA, et al. Transcatheter thrombolytic therapy for acute mesenteric and portal vein thrombosis. J Vasc Interv Radiol 2005; 16: 651-661.

17. Blum U, Haag K, Rossle M, et al. Noncavernomatous portal vein thrombosis in hepatic cirrhosis: treatment with transjugular intrahepatic portosystemic shunt and local thrombolysis. Radiology 1995; 195: 153-157.

18. Kercher KW, Sing RF, Watson KW, et al. Transhepatic thrombolysis in acute portal vein thrombosis after laparoscopic splenectomy. Surg Laparosc Endosc Percutan Tech 2002; 12: 131-136.

19. Bilbao JI, Longo JM, Rousseau H, et al. Transjugular intrahepatic portocaval shunt after thrombus disruption in partially thrombosed portal veins. Cardiovasc Intervent Radiol 1994; 17: 106-109.

20. Haskal ZJ. Power-pulse thrombolysis, thrombectomy, and TIPS formation for the accelerated treatment of portosplenomesenteric thrombosis in Budd-Chiari syndrome. J Vasc Interv Radiol 2007; 18: 1458-1460.

21. Wichman HJ, Cwikiel W, Keussen I. Interventional treatment of mesenteric venous occlusion. Pol J Radiol 2014; 79: 233-238.

22. Valla D. Splanchnic vein thrombosis. Semin Thromb Hemost 2015; 41: 494-502.

23. Pereira K, Carrion AF, Martin P, et al. Current diagnosis and management of post-transjugular intrahepatic portosystemic shunt refractory hepatic encephalopathy. Liver Int 2015; 35: 2487-2494.

24. Chen B, Wang W, Tam MD, et al. Transjugular intrahepatic portosystemic shunt in liver transplant recipients: indications, feasibility, and outcomes. Hepatol Int 2015; 9: 391-398.

25. Senzolo M, Tibbals J, Cholongitas E, et al. Transjugular intrahepatic portosystemic shunt for portal vein thrombosis with and without cavernous transformation. Aliment Pharmacol Ther 2006; 23: 767-775.

26. Salem R, Vouche M, Baker T, et al. Pretransplant portal vein recanalization-transjugular intrahepatic portosystemic shunt in patients with complete obliterative portal vein thrombosis. Transplantation 2015; 99: 2347-2355.

27. Han G, Qi X, He C, et al. Transjugular intrahepatic portosystemic shunt for portal vein thrombosis with symptomatic portal hypertension in liver cirrhosis. J Hepatol 2011; 54: 78-88.

28. Chawla YK, Bodh V. Portal vein thrombosis. J Clin Exp Hepatol 2015; 5: 22-40.

29. Trebicka J, Strassburg CP. Etiology and complications of portal vein thrombosis. Viszeralmedizin 2014; 30: 375-380.

30. Amitrano L, Guardascione MA, Scaglione M, et al. Prognostic factors in noncirrhotic patients with splanchnic vein thromboses. Am J Gastroenterol 2007; 102: 2464-2470. 